\title{
A Comparison of Reflectance and Attenuated Total Internal Reflection Infrared Microspectroscopic Imaging Techniques for the Analysis of Kidney Stones.
}

\author{
Chen Ling ${ }^{1}$, James C. Williams $\mathrm{Jr}^{2}$, Andrew P. Evan ${ }^{2}$ and André J. Sommer ${ }^{1}$ \\ 1. Molecular Microspectroscopy Laboratory, Department of Chemistry and Biochemistry/Miami \\ University, Oxford, $\mathrm{OH} 45056$, USA \\ 2. Department of Anatomy and Cell Biology/Indiana University School of Medicine, Indianapolis, IN \\ 46202, USA
}

Kidney stone disease, also known as urolithiasis, is common throughout the world. The causes of kidney stone disease are still uncertain and numerous studies have been carried out to determine the formation factors of the disease. The most prevalent constituent in kidney stones is calcium oxalate, which appears in about $80 \%$ of the cases, while hydroxyl apatite is the second most frequent constituent, appearing in approximately $12 \%$ of the cases [1]. In order to determine the causes of kidney stone development, two approaches can be taken. First, the very onset of stone formation in the tissue can be studied in tissue biopsies. In this case the chemical identity of micrometer sized mineral inclusions and their surroundings are studied. Second, cross-sectioned stones allow one to study the deposition/formation of the stone as a function of time much like geologists study sedimentary rock formations.

Many analytical methods have been applied in each approach but those methods that maintain the integrity of the sample and provide molecular information (unique identification) as well as spatial information offer the best chance of elucidating the formation mechanisms. Over the past several years we have shown that reflectance infrared imaging has been useful in studying cross-sectioned stones and more recently that attenuated total internal reflection (ATR) infrared imaging has been useful for studying mineral inclusions in tissue biopsies [2-4]. In the present study we compare the capabilities and limitations of each method to study cross-sectioned stones.

In reflectance imaging, infrared light reflected by the sample is studied as a function of vibrational energy. However, one difficulty using this method is that the reflected light contains both reflection information and absorption information. Depending on the morphology of the sample, separating these different properties can be difficult. In addition, the amount of reflected light is relatively low unless the material exhibits Reststrahlen bands [4].

ATR infrared imaging is, by its very nature an absorption method which all but eliminates the reflection information. In this method a germanium hemisphere is placed in intimate contact with the sample. Infrared light evanescently penetrates the sample where it can be absorbed thereby providing molecular information. The method has been shown to eliminate many problems associated with both transflectance and reflectance measurements. One additional benefit is that the hemisphere provides an optical immersion effect, which increases the spatial resolution of the method by a factor of four over reflectance infrared imaging [5].

Kidney stones ranging in size from 2 to $7 \mathrm{~mm}$ in diameter were embedded in methyl methacrylate resin and cross-sectioned to a thickness of $\sim 1 \mathrm{~mm}$ using a diamond wire saw. Infrared images of the sectioned stones in were collected with a Perkin-Elmer Spectrum Spotlight 300 IR imaging microscope using a linear array $\mathrm{HgCdTe}(\mathrm{MCT})$ detector. Each pixel on the detector represents $6.25 \times 6.25 \mu \mathrm{m}^{2}$ area on the sample for the reflectance image and a $1.56 \mathrm{X} 1.56 \mu \mathrm{m}^{2}$ area for the ATR image. Spectra were collected at a spectral resolution of $4 \mathrm{~cm}^{-1}$ and represent the average of 2 coadded scans. A principle 
component regression analysis was conducted on the images to discern the number of different spectra components making up the images.

Figure 1 illustrates reflected white light, infrared reflectance and ATR images of the section. Clearly the ATR image has much better detail owing to the increased spatial resolution of the method. The lack of clarity in the reflectance image is due not only to poorer resolution, but also scattering and the mixing effects cited earlier. Spectra extracted from each image are shown in Figure 1. The spectrum from the ATR image more closely approximates a traditional transmission spectrum allowing easier interpretation. At present, the only limitation of the ATR method is the field of view. However, Patterson has shown by increasing the diameter of the hemisphere the field of view can be increased to $2.5 \mathrm{X} 2.5 \mathrm{~mm}$ [6].

\section{References:}

[1] S. R. Khan, Urol. Res. 34 (2006) p. 86

[2] J. C. Anderson et al, Urol. Res. 35 (2007) p. 41-48

[3] H. J. Gulley-Stahl, and A. J. Sommer in "Evanescent Wave Imaging”, ed. G. Srinivasan, (McGrawHill Companies, Inc, China) p. 100-102

[4] H. J. Gulley-Stahl et al, Appl. Spectrosc. 64(1) (2010) p.15-22

[5] Tisinger et al, Appl. Spectrosc. 55 (3) (2001) p.252-256

[6] Patterson et al, Appl. Spectrosc. 60 (11) (2006) p.1256-1266
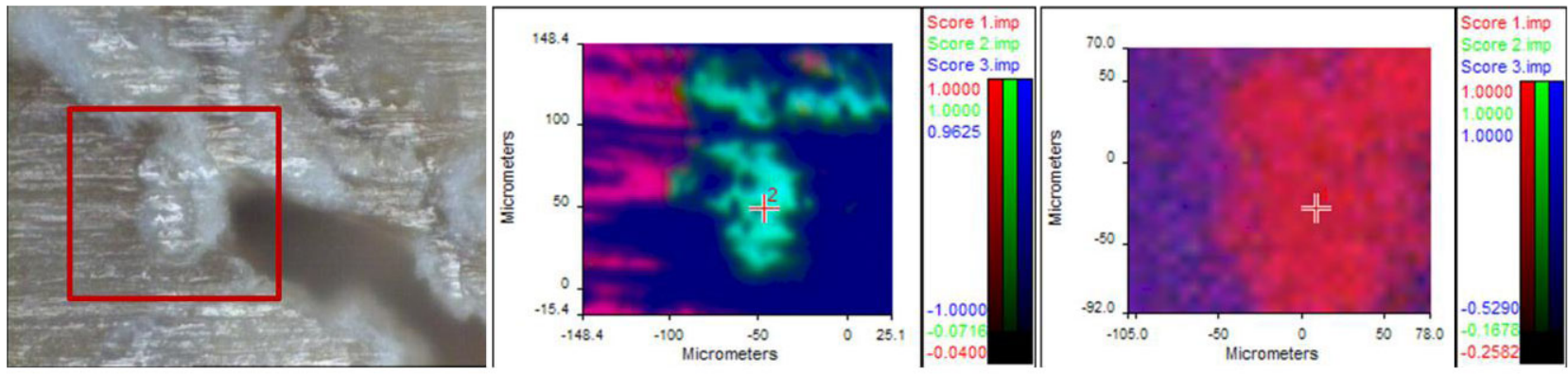

Figure 1. Visible image (left), ATR image (middle), and reflectance image (right) maps of the stone cross-section (shown in the red box in visible image). Spectra to the right were extracted from the ATR image and Reflectance image at the same spot as shown. The feature near $1020 \mathrm{~cm}^{-1}$ is due to asymmetric stretch of orthophosphate group in apatite.

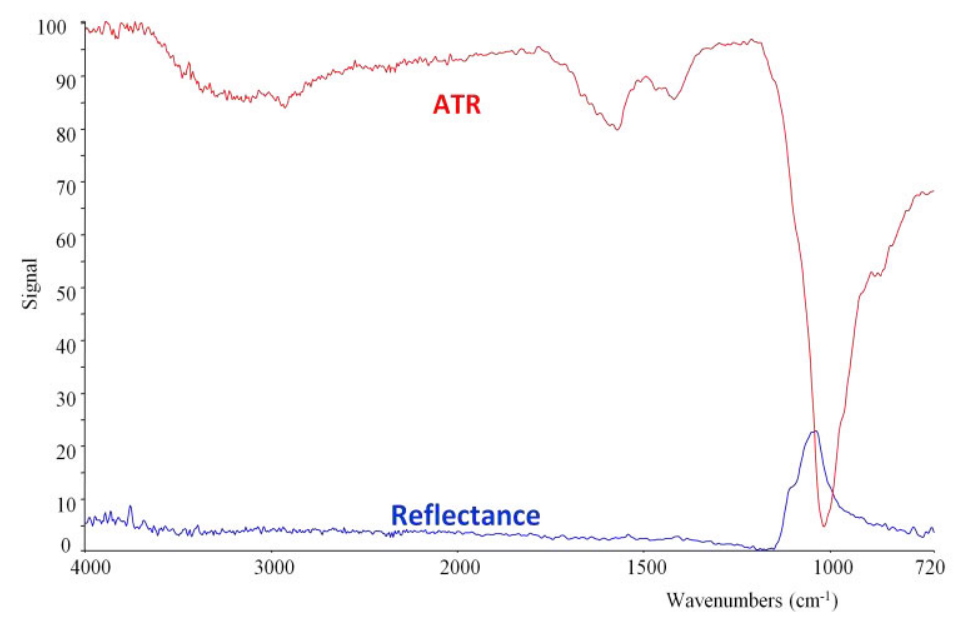

